\title{
Enantioselective Partial Reduction of 2,5-Disubstituted Pyrroles via a Chiral Protonation Approach
}

\author{
Timothy J. Donohoe*, Catherine E. Headley, Caroline L. Rigby, \\ Gurdip Bhalay and Rick P. C. Cousins
}

\section{Supplementary Material}

\begin{abstract}
General
Analytical HPLC was used for the determination of enantiomeric excess using a Waters 600E System Controller, a Waters 991 Photodiode Array detector and a Daicel Chiralpak AD column.
\end{abstract}

\section{Chiral protonation procedure}

Lithium (25 mg, $36 \mathrm{mmol})$ and di-tert-butyl biphenyl (19 mg, $0.075 \mathrm{mmol})$ were placed in a Schlenk tube containing anti-bumping granules under an atmosphere of argon and left to grind for three hours. THF ( $25 \mathrm{ml})$ was added and the resulting blue solution was cooled to $-78^{\circ} \mathrm{C}$, a solution of $\mathbf{1}(100 \mathrm{mg}, 0.36 \mathrm{mmol})$ in $\mathrm{THF}(5 \mathrm{ml})$ was then added dropwise and the mixture was allowed to stir until the return of the blue colour. 1,2-dibromoethane $(0.2 \mathrm{ml})$ was added to the mixture followed by a solution of ephedrine (144 mg, $0.9 \mathrm{mmol})$ in THF $(5 \mathrm{ml})$, after 5 minutes the reaction was quenched with saturated ammonium chloride $(5 \mathrm{ml})$ and allowed to warm to room temperature. The crude reaction mixture was extracted into ether $(2 \times 30 \mathrm{ml})$, washed with $1 \mathrm{M} \mathrm{HCl}(30 \mathrm{ml})$ and dried over $\mathrm{MgSO}_{4}$ before being filtered and concentrated in vacuo to give a 1:1 mixture of trans-2 and cis-2 (86 mg, 84\%) which were separable by flash column chromatography (eluting with a 4:1 mixture of petrol:EtOAc) yielding trans-2 (43 mg, 42\%, 74\% ee) as a white crystalline solid and cis-2 (43 mg, $42 \%)$ as a yellow oil. 
cis-2,5-dihydro-pyrrole-1,2,5-tricarboxylic acid tert-butyl ester dimethyl ester, cis-2

$v_{\max } / \mathrm{cm}^{-1} 2977,2955,1763,1739,1710 ; \delta_{\mathrm{H}}\left(300 \mathrm{MHz}, \mathrm{CDCl}_{3}\right) 1.43\left(\mathrm{~s}, 9 \mathrm{H}, \mathrm{C}\left(\mathrm{CH}_{3}\right)_{3}\right)$, $3.79\left(\mathrm{~s}, 6 \mathrm{H}, 2 \mathrm{x} \mathrm{CO}_{2} \mathrm{CH}_{3}\right), 5.06(\mathrm{dt}, 1 \mathrm{H}, J 2.6,2.1, \mathrm{NCHCH}=\mathrm{CH}), 5.12(\mathrm{dt}, 1 \mathrm{H}, J 2.5$, 2.1, $\mathrm{NCHCH}=\mathrm{CH}), 5.96(\mathrm{ddt}, 2 \mathrm{H}, J 14,6.2,2.1, \mathrm{NCH} H \mathrm{C}=\mathrm{CH}) ; \delta_{\mathrm{H}}(300 \mathrm{MHz}$, DMSO, $\left.140{ }^{\circ} \mathrm{C}\right) 1.41\left(\mathrm{~s}, 9 \mathrm{H}, \mathrm{C}\left(\mathrm{CH}_{3}\right)_{3}\right), 3.53\left(\mathrm{~s}, 6 \mathrm{H}, 2 \mathrm{x} \mathrm{CO}_{2} \mathrm{CH}_{3}\right), 5.01(\mathrm{~m}, 2 \mathrm{H}$, $\left.\mathrm{NCHCO}_{2} \mathrm{CH}_{3}\right), 5.96(\mathrm{~m}, 2 \mathrm{H}, \mathrm{HC}=\mathrm{CH}) ; \delta_{\mathrm{C}}\left(75 \mathrm{MHz}, \mathrm{CDCl}_{3}\right)$ 28.1, 52.3, 52.4, 66.5, 66.9, 90.0, 127.4, 127.5, 152.9, 169.5; MS (CI, $m / z) 286(\mathrm{M}+1,10 \%)$ and $186(\mathrm{M}$ -99, $100 \%$ ); HRMS (CI, $m / z$ ) found M 286.1294, $\mathrm{C}_{13} \mathrm{H}_{20} \mathrm{NO}_{6}$ requires $M$ 286.1291.

trans-2,5-dihydro-pyrrole-1,2,5-tricarboxylic acid tert-butyl ester dimethyl ester, trans-2

$\mathrm{mp}\left({ }^{\circ} \mathrm{C}\right)$ 64-66; $\mathrm{u}_{\max } / \mathrm{cm}^{-1} 2976,1756,1711 ; \delta_{\mathrm{H}}\left(400 \mathrm{MHz}, \mathrm{CDCl}_{3}\right) 1.44(\mathrm{~s}, 9 \mathrm{H}$, $\left.\mathrm{C}\left(\mathrm{CH}_{3}\right)_{3}\right), 3.75\left(\mathrm{~s}, 3 \mathrm{H}, \mathrm{CO}_{2} \mathrm{CH}_{3}\right), 3.76\left(\mathrm{~s}, 3 \mathrm{H}, \mathrm{CO}_{2} \mathrm{CH}_{3}\right), 5.11(\mathrm{dt}, 1 \mathrm{H}, J$ 6.0, 2.0 $\mathrm{NCHCH}=\mathrm{CH}), 5.19(\mathrm{dt}, 1 \mathrm{H}, J$ 6.0, $2.0 \mathrm{NCHCH}=\mathrm{CH}), 5.87(\mathrm{dt}, 1 \mathrm{H}, J$ 6.8, 2.0 $\mathrm{NCHCH}=\mathrm{CH}), 5.92(\mathrm{dt}, 1 \mathrm{H}, J 6.0,2.0 \mathrm{NCHCH}=\mathrm{CH}) ; \delta_{\mathrm{H}}\left(300 \mathrm{MHz}, \mathrm{DMSO}, 140{ }^{\circ} \mathrm{C}\right)$ $1.38\left(\mathrm{~s}, 9 \mathrm{H}, \mathrm{C}\left(\mathrm{CH}_{3}\right)_{3}\right), 3.68$ (s, 6H, 2x CO $\left.\mathrm{CH}_{3}\right), 5.00(\mathrm{~m}, 2 \mathrm{H}, 2 \mathrm{x} \mathrm{NCHCH}=\mathrm{CH}), 5.98$ $(\mathrm{m}, 2 \mathrm{H}, \mathrm{NCHCH}=\mathrm{CH}) ; \delta_{\mathrm{C}}\left(75 \mathrm{MHz}, \mathrm{CDCl}_{3}\right)$ 28.0, 52.2, 52.3, 66.6, 66.9, 81.0, 127.8, 127.9, 152.6, 169.7, 170.2; MS (CI, m/z) 286 (M +1, 10\%), 247 ( $\left.\mathrm{M}^{-} 38,100 \%\right), 230$

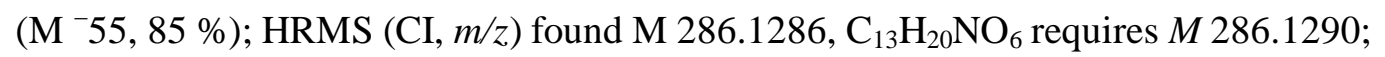
Elemental analysis found $\mathrm{C}, 54.68, \mathrm{H}, 6.57, \mathrm{~N}, 4.85 \mathrm{C}_{13} \mathrm{H}_{19} \mathrm{NO}_{6}$ requires $\mathrm{C}, 54.73, \mathrm{H}$, $6.71, \mathrm{~N}, 4.91$.

\section{Partial quenching experiments}

Lithium (25 mg, $36 \mathrm{mmol})$ and di-tert-butyl biphenyl (19 mg, $0.075 \mathrm{mmol})$ were placed in a Schlenk tube containing anti-bumping granules under an atmosphere of Argon and left to grind for three hours. THF ( $25 \mathrm{ml})$ was added and the resulting blue solution was cooled to $-78^{\circ} \mathrm{C}$, a solution of $\mathbf{1}(100 \mathrm{mg}, 0.36 \mathrm{mmol})$ in $\mathrm{THF}$ was then added dropwise and the mixture was allowed to stir until the return of the blue colour. 1,2-dibromoethane $(0.2 \mathrm{ml})$ was added to the mixture followed by a solution of 
ephedrine $(59 \mathrm{mg}, 0.36 \mathrm{mmol})$ in $\mathrm{THF}(5 \mathrm{ml})$, after 5 minutes the reaction was quenched with saturated ammonium chloride $(5 \mathrm{ml})$ and allowed to warm to room temperature. The crude reaction mixture was extracted into ether $(2 \times 30 \mathrm{ml})$, washed with $1 \mathrm{M} \mathrm{HCl}(30 \mathrm{ml})$ and dried over $\mathrm{MgSO}_{4}$ before being filtered and concentrated in vacuo to give a 1:1 mixture of trans-2 and cis-2 (41 mg, 40\%) which were separable by flash column chromatography (eluting with a 4:1 mixture of petrol:EtOAc) yielding trans-2 (20 mg, 20\%, 45\% ee) as a white crystalline solid and cis-2 (20 mg, $20 \%$ ) as a yellow oil.

\section{Recrystallisation}

Trans-2 (290 mg, $1 \mathrm{mmol})$ with an ee of $74 \%$ was recrystallised from light petroleum:ether giving product $(210 \mathrm{mg}, 0.7 \mathrm{mmol})$ with an ee of $94 \%$. $[\alpha]_{\mathrm{D}}^{23}:+391$ (c $1, \mathrm{CHCl}_{3}$ ). Melting point of recrystallised material: $68-72^{\circ} \mathrm{C}$.

\section{Hydrogenation of double bond}

Palladium on charcoal was added to a solution of enantiomerically pure trans-2 (50 $\mathrm{mg}, 0.18 \mathrm{mmol})$ in EtOH $(2 \mathrm{ml})$. The mixture was stirred under an atmosphere of hydrogen for 18 hours. It was then filtered through Celite and the solvent was removed in vacuo to yield a colourless oil $(52 \mathrm{mg}, 100 \%)$. $[\alpha]_{\mathrm{D}}^{23}:+63\left(\mathrm{c} 1, \mathrm{CHCl}_{3}\right)$. This value was compared to the optical rotation reported in the literature for this compound. ${ }^{1}$

$\delta_{\mathrm{H}}\left(200 \mathrm{MHz}, \mathrm{CDCl}_{3}\right) 1.42\left(\mathrm{~s}, 9 \mathrm{H}, \mathrm{C}\left(\mathrm{CH}_{3}\right)_{3}\right), 1.95-2.02\left(\mathrm{~m}, 2 \mathrm{H}, \mathrm{CH}_{2} \mathrm{CH}_{2}\right), 2.27-2.38$ (m, 2H, $\left.\mathrm{CH}_{2} \mathrm{CH}_{2}\right), 3.72\left(\mathrm{~s}, 6 \mathrm{H}, \mathrm{CO}_{2} \mathrm{CH}_{3}\right), 4.38-4.58(\mathrm{~m}, 2 \mathrm{H}, \mathrm{NCHCH}=\mathrm{CH})$.

\footnotetext{
${ }^{1}$ Sasaki N. A.; Sagnard I. Tetrahedron. 1994, 50, 7093.
} 